\title{
Numerical Solution of the Rotating Shallow Water Flows with Topography Using the Fractional Steps Method
}

\author{
Hossam S. Hassan ${ }^{1}$, Khaled T. Ramadan ${ }^{1}$, Sarwat N. Hanna ${ }^{2}$ \\ ${ }^{1}$ Department of Basic and Applied Science, Arab Academy for Science, \\ Technology and Maritime Transport, Alexandria, Egypt \\ ${ }^{2}$ Department of Engineering Mathematics and Physics, Faculty of Engineering, \\ Alexandria University, Alexandria, Egypt \\ E-mail: hossams@aast.edu \\ Received April 16, 2010; revised June 2, 2010; accepted June 12, 2010
}

\begin{abstract}
The two-dimensional nonlinear shallow water equations in the presence of Coriolis force and bottom topography are solved numerically using the fractional steps method. The fractional steps method consists of splitting the multi-dimensional matrix inversion problem into an equivalent one dimensional problem which is successively integrated in every direction along the characteristics using the Riemann invariant associated with the cubic spline interpolation. The height and the velocity field of the shallow water equations over irregular bottom are discretized on a fixed Eulerian grid and time-stepped using the fractional steps method. Effects of the Coriolis force and the bottom topography for particular initial flows on the velocity components and the free surface elevation have been studied and the results are plotted.
\end{abstract}

Keywords: Shallow Water Equations, Fractional Steps Method, Riemann Invariants, Bottom Topography, Cubic Spline Interpolation

\section{Introduction}

Shallow water equations form a set of hyperbolic partial differential equations that describe the flow below the pressure surface in the fluid, sometimes, but not necessarily, a free surface. The equations are derived from depth-integrating the Navier-Stokes equations, in the case where the horizontal length scale is much greater than the vertical length scale. They can be used to model Rossby and Kelvin waves in the atmosphere, rivers, lakes and oceans in a large domain as well as gravity waves. The rotating shallow water equations including topographic effects are a leading order model to study coastal hydrodynamics on several scales including intermediate scale rotational waves and breaking waves on beaches. Also, they are used with Coriolis forces in atmospheric and oceanic modeling, as a simplification of the primitive equations of atmospheric flow.

Due to the nonlinearity of the model as well as the complexity of the geometries encountered in real-life applications such as flow of pollutants, tsunamis, avalanches, dam break, flooding, potential vorticity field...etc, much effort has been made in recent years to develop numerical methods to solve the equations approximately.

Bottom topography plays a major role in determining the flow field in the oceans, rivers, shores, coastal sea and so on. One of the most important applications of the shallow water waves is the tsunami waves [1], usually generated by underwater earthquakes which cause an irregular topography of increasing or decreasing water depth. In particular, the main problem in solving the shallow water equations is the presence of the source terms modeling the bottom topography and the Coriolis forces included in the system.

The shallow water equations used in geophysical fluid dynamics are based on the assumption $\mathrm{H} / \mathrm{L}<<1$, where $\mathrm{H}$ and $\mathrm{L}$ are the characteristic values for the vertical and horizontal length scales of motion respectively. These equations are a two-dimensional hyperbolic system modeling the depth and the depth-averaged horizontal velocities for an incompressible fluid.

Perhaps, rotation is the most important factor that distinguishes geophysical fluid dynamics from classical fluid dynamics. If latitudinal varying Coriolis forces are included in the shallow water equations, the resulting system can support both Rossby and gravity waves. On the other hand, by neglecting the Coriolis forces in the shal- 
low water system, there is no Rossby-wave solution for the system.

The Coriolis force is proportional in magnitude to the flow speed and directed perpendicular to the direction of the flow. It acts to the left of the flow in the southern hemisphere and to the right in the northern hemisphere. A somewhat inaccurate but helpful way to see why the direction is different in the two hemispheres is related to the principle of conservation of angular momentum. For a given horizontal motion, the strongest horizontal deflection is at the poles and there is no horizontal deflection at the equator; for vertical motion the opposite is true. The magnitude of the Coriolis force proportionally depends upon the latitude and the wind speed. The direction of the Coriolis force always acts at right angles to the direction of movement, which is to the right in the Northern Hemisphere and to the left in the Southern Hemisphere [2].

Many authors have used different numerical techniques to solve the shallow water equations such as finite volume method, finite element method and fractional steps method.

Lukácová-Medvid'ová et al. [3] presented a new wellbalanced finite volume method within the framework of the finite volume evolution Galerkin (FVEG) schemes for the shallow water equations with source terms modeling the bottom topography and the Coriolis forces.

Gallouët et al. [4] studied the computation of the shallow water equations with topography by finite volume method, in a one-dimensional framework. In their paper, they considered approximate Riemann solvers. Several single step methods are derived from this formulation and numerical results are compared with the fractional steps method.

Dellar and Salmon [5] derived an extended set of shallow water equations that describe a thin inviscid fluid layer above fixed topography in a frame rotating about an arbitrary axis.

Karelsky et al. [6] executed the generalization of classical shallow water theory to the case of flows over an irregular bottom. They showed that the simple self-similar solutions that are characteristic for the classical problem exist only if the underlying surface has a uniform slope.

George [7] presented a class of augmented approximate Riemann solvers for the one-dimensional shallow water equations in the presence of an irregular bottom, neglecting the effect of Coriolis force. These methods belong to the class of finite volume Godunov type methods that use a set of propagation jump discontinuities, or waves, to approximate the true Riemann solution.

Shoucri [8] applied the fractional steps technique for the numerical solution of the shallow water equations with flat bottom in the presence of the Coriolis force. The method of fractional steps that he presented in his paper has the great advantage of solving the shallow wa- ter equations without the iterative steps involved in the multi-dimensional interpolation, and without the iteration associated with the intermediate step of solving the Helmholtz equation [9].

Abd-el-Malek and Helal [10] developed a mathematical simulation to determine the water velocity in the Lake Mariut, taking into consideration its concentration and the distribution of the temperature along it, by applying the fractional steps method for the numerical solution of the shallow water equations.

Shoucri [11] applied the fractional steps technique for the numerical solution of the shallow water equations to study the evolution of the vorticity field. The method is Eulerian [8], and the different variables are discretized on a fixed grid.

Yohsuke et al. [12] presented two efficient explicit schemes with no iterative process for the two-dimensional shallow-water equations of a hydrostatic weather forecast model. One is the directional-splitting fractional-step method, which uses a treatment based on the characteristics approach (Shoucri $[8,11]$ ). The other is the interpolated differential operator (IDO) scheme (Aoki [13]), which is one of the multimoment Eulerian schemes. They compared the forecast geopotential heights obtained from the fractional-step method and the IDO scheme after $48 \mathrm{~h}$ for various resolutions with those of the referenced scheme by Temperton and Staniforth [14].

Rotating shallow water equations including topographic terms are numerically dealt with the fractional steps method. In most real applications there is variable bottom topography that adds a source term to the shallow water equations. There are several works, where both the Coriolis forces as well as the bottom topography are taken into account, see [3,15-17]. A standard and easy way to deal with these source terms is to treat them independently by using the fractional steps method. It has the great advantage of solving the equations without the iterative steps involved in the multidimensional interpolation problems.

In this work, we apply the fractional steps method to solve the two-dimensional shallow water equations with source terms (including the Coriolis force and bottom topography) for different initial flows observed in the real-life such as the tsunami propagation wave and the dam break wave. The objective of the present work is to simulate the influence of different profiles of the irregular bottom in case of neglecting and including the effect of the Coriolis force on the velocity component in the $\mathrm{x}$-and $\mathrm{y}$-directions, water depth and the free surface elevation for different time. The results are illustrated graphically for particular initial flows.

\section{Mathematical Formulation of the Problem}

The shallow water equations (Saint-Venant equations) 
describe the free surface flow of incompressible water in response to gravitational and rotational accelerations (Coriolis accelerations), where the vertical depth of water is much less than the horizontal wavelength of the disturbance of the free surface [wave motion]. These equations are often used as a mathematical model when numerical methods for solving weather or climate prediction problems are tested. Figure 1 illustrates the shallow water model where, " $h$ " denotes the water height above the non-flat bottom, " $H$ " is the undisturbed free surface level, $\zeta=\zeta(x, y)$ denotes the bottom topography, " $h *$ " is the water height above the flat bottom and $\eta=h^{*}-H$ denotes the free surface elevation.

The Continuity equation and the momentum equations for the two-dimensional shallow water equations system taking into account the effects of topography and the Earth's rotation are formulated by Pedlosky [2] as

Continuity equation:

$$
\frac{\partial h}{\partial t}+\frac{\partial(u h)}{\partial x}+\frac{\partial(v h)}{\partial y}=0
$$

Momentum equations:

$$
\begin{aligned}
& \frac{\partial(h u)}{\partial t}+\frac{\partial\left(h u^{2}\right)}{\partial x}+\frac{\partial(h u v)}{\partial y} \\
& +\frac{g}{2} \frac{\partial\left(h^{2}\right)}{\partial x}=-g h \frac{\partial \zeta}{\partial x}+f h v \\
& \frac{\partial(h v)}{\partial t}+\frac{\partial(h u v)}{\partial x}+\frac{\partial\left(h v^{2}\right)}{\partial y} \\
& +\frac{g}{2} \frac{\partial\left(h^{2}\right)}{\partial y}=-g h \frac{\partial \zeta}{\partial y}-f h u
\end{aligned} .
$$

The model is run over a rectangular domain centered at the earth origin, such that the x-axis is taken eastward and the y-axis is taken northward, with $u$ and $v$ the corresponding velocity components, respectively, g stands for the gravitational constant, $f=2 \Omega \sin \phi$ is the Coriolis parameter, $\Omega$ is the angular velocity of the earth rotation, $\phi$ is the geographical latitude of the earth origin coordinate and $(f v,-f u)$ represents the Coriolis

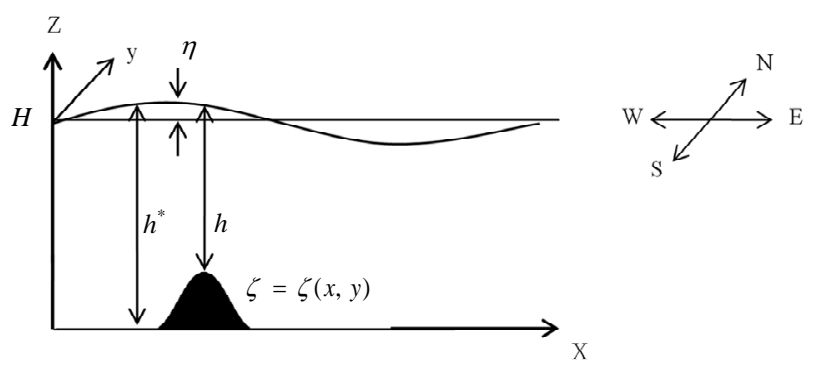

Figure 1. Geometry of the shallow water model. acceleration which is produced by the effect of rotation.

Let the geopotential height be

$$
\Phi=g h
$$

Substitution (4) into (1)-(3), yields

$$
\begin{aligned}
& \frac{\partial \Phi}{\partial t}+u \frac{\partial \Phi}{\partial x}+\Phi \frac{\partial u}{\partial x}+v \frac{\partial \Phi}{\partial y}+\Phi \frac{\partial v}{\partial y}=0 \\
& \frac{\partial u}{\partial t}+u \frac{\partial u}{\partial x}+v \frac{\partial u}{\partial y}+\frac{\partial \Phi}{\partial x}=-g \frac{\partial \zeta}{\partial x}+f v \\
& \frac{\partial v}{\partial t}+u \frac{\partial v}{\partial x}+v \frac{\partial v}{\partial y}+\frac{\partial \Phi}{\partial y}=-g \frac{\partial \zeta}{\partial y}-f u
\end{aligned}
$$

We concern ourselves to approximate the two dimensional shallow water Equations (5)-(7) with source terms modelling the bottom topography and the Coriolis forces for different initial flows observed in the real-life such as the tsunami propagation wave and the dam break wave.

\section{Solution of the Problem}

Our solution is based on applying the fractional steps method which was first proposed by Yanenko [18], to the system of non-linear partial differential Equations (5)-(7) by splitting the equations into two one-dimensional problems that are solved alternately in $\mathrm{x}$ - and $\mathrm{y}$ directions [19].

\subsection{Fractional Steps Method}

Step 1. Solve for $\Delta t / 2$ in the $x$-direction (without the source terms):

Equations (5)-(7) will be

$$
\begin{gathered}
\frac{\partial \Phi}{\partial t}+u \frac{\partial \Phi}{\partial x}+\Phi \frac{\partial u}{\partial x}=0, \\
\frac{\partial u}{\partial t}+u \frac{\partial u}{\partial x}+\frac{\partial \Phi}{\partial x}=0, \\
\frac{\partial v}{\partial t}+u \frac{\partial v}{\partial x}=0 .
\end{gathered}
$$

Equations (8) and (9) can be written as

$$
\frac{\partial R_{x \pm}}{\partial t}+(u \pm \sqrt{\Phi}) \frac{\partial R_{x \pm}}{\partial x}=0
$$

where $R_{x \pm}=u \pm 2 \sqrt{\Phi}$ are the Riemann invariants.

By applying the classical finite difference scheme to (11), which is a first-order equation in time and space, we get 


$$
\begin{aligned}
& {\left[R_{x \pm}(x, y, t+\Delta t / 2)-R_{x \pm}(x, y, t)\right]} \\
& +(u \pm \sqrt{\Phi}) \Delta t /(2 \Delta x) \\
& {\left[R_{x \pm}(x+\Delta x, y, t)-R_{x \pm}(x, y, t)\right]=0 .}
\end{aligned}
$$

The CFL stability condition is given by $\mid(u \pm \sqrt{\Phi})$ $\Delta t /(2 \Delta x) \mid \leq 1$. Let $\Delta x=-(u \pm \sqrt{\Phi}) \Delta t / 2$, so, (12) will be

$$
\begin{aligned}
& R_{x \pm}(x, y, t+\Delta t / 2) \\
= & R_{x \pm}(x-(u \pm \sqrt{\Phi}) \Delta t / 2, y, t)
\end{aligned}
$$

The right-hand side of (13) is the value of the function at time $t=n \Delta t$ at the departure points of the characteristics. The value of the function at $t+\Delta t / 2$ at the arrival grid points is obtained using cubic spline interpolation from the values of the function at the grid points at $t=n \Delta t$, (i.e. the value $R_{x \pm}$ after the time $\Delta t / 2$ from time $\mathrm{t}$ is the same value of $R_{x \pm}$ at that same time but after the distance $x$ is shifted by $\Delta x$ ).

Similarly, the solution of (10) for $\mathrm{v}$ at $t+\Delta t / 2$ is written as

$$
v(x, y, t+\Delta t / 2)=v(x-u \Delta t / 2, y, t),
$$

where $\Delta x=-u \Delta t / 2$, i.e. the solution of Equations (8)-(10) can be written after time $(n+1 / 2) \Delta t, \mathrm{n}=$ $1,2,3 \ldots$ as the equality

$$
\left(\begin{array}{c}
R_{x \pm} \\
v
\end{array}\right)_{(x, y,(n+1 / 2) \Delta t)}=\left(\begin{array}{c}
R_{x \pm} \\
v
\end{array}\right)_{\left(x_{ \pm}, y, n \Delta t\right)}
$$

where $x_{ \pm}=x-(u \pm \sqrt{\Phi}) \Delta t / 2$ for the first row and $x_{ \pm}=x-u \Delta t / 2$ for the second row. Hence the CFL stability condition will be satisfied automatically at any time evolution $(n+1 / 2) \Delta t$.

So, The solutions of $R_{x \pm}$, give the values of $\mathrm{h}$ and $\mathrm{u}$ after time $(n+1 / 2) \Delta t$ in the $x$-direction. So, the interpolated values in (13) and (14) are calculated using the cubic spline interpolation, where no iteration is implied in this calculation.

Step 2. Solve for $\Delta t / 2$ in the $y$-direction (without the source terms):

Equations (5)-(7) will be

$$
\begin{gathered}
\frac{\partial \Phi}{\partial t}+v \frac{\partial \Phi}{\partial y}+\Phi \frac{\partial v}{\partial y}=0 \\
\frac{\partial u}{\partial t}+v \frac{\partial u}{\partial y}=0
\end{gathered}
$$

$$
\frac{\partial v}{\partial t}+v \frac{\partial v}{\partial y}+\frac{\partial \Phi}{\partial y}=0
$$

Use the results obtained at the end of Step 1, to solve (15)-(17) for $\Delta t / 2$.

Equations (15) and (17) can be rewritten as

$$
\frac{\partial R_{y \pm}}{\partial t}+(v \pm \sqrt{\Phi}) \frac{\partial R_{y \pm}}{\partial y}=0,
$$

where $R_{y \pm}=v \pm 2 \sqrt{\Phi}$ are the Riemann invariants.

The solutions of (16) and (18) at $t+\Delta t / 2$ are

$$
u(x, y, t+\Delta t / 2)=u(x, y-v \Delta t / 2, t) \text {, }
$$

and

$$
\begin{aligned}
& R_{y \pm}(x, y, t+\Delta t / 2) \\
= & R_{y \pm}(x, y-(v \pm \sqrt{\Phi}) \Delta t / 2, t),
\end{aligned}
$$

i.e. after time $(n+1 / 2) \Delta t$ we rewrite Equations (19) and (20) as

$$
\left(\begin{array}{c}
R_{y \pm} \\
u
\end{array}\right)_{(x, y,(n+1 / 2) \Delta t)}=\left(\begin{array}{c}
R_{y \pm} \\
u
\end{array}\right)_{\left(x, y_{ \pm}, n \Delta t\right)}
$$

where $y_{ \pm}=y-(v \pm \sqrt{\Phi}) \Delta t / 2$ for the first row and $y_{ \pm}=y-v \Delta t / 2$ for the second row.

Again, the solutions of $R_{y \pm}$, give the values of $h$ and $v$ after $\Delta t / 2$ in the y-direction.

Step 3. Solve for $\Delta t$ (for the source terms):

Equations (5)-(7) will be

$$
\begin{aligned}
& \frac{\partial u}{\partial t}=-g \frac{\partial \zeta}{\partial x}+f v \\
& \frac{\partial v}{\partial t}=-g \frac{\partial \zeta}{\partial y}-f u
\end{aligned}
$$

Use the results obtained at the end of Step 2, to solve (21) and (22) for $\Delta t$.

Solutions of (21) and (22) are calculated at $\Delta t$

$$
\begin{aligned}
& u(x, y,(n+1) \Delta t) \\
= & U_{0}-(g \Delta t / \Delta x)[\zeta(x, y, n t) \\
& -\zeta(x-\Delta x, y, n t)]+f V_{0} \\
& v(x, y,(n+1) \Delta t) \\
= & V_{0}-(g \Delta t / \Delta y)[\zeta(x, y, n t) \\
& -\zeta(x, y-\Delta y, n t)]-f U_{0}
\end{aligned}
$$

where $U_{0}$ and $V_{0}$ are the values of $u$ and $v$ at the beginning of Step 3.

Step 4. Repeat Step 2: 
The results obtained at the end of Step 3 are used to solve for $\Delta t / 2$ the equations in the y-direction as in Step 2.

$$
\text { i.e. }\left(\begin{array}{c}
R_{y \pm} \\
u
\end{array}\right)_{(x, y,(n+1) \Delta t)}=\left(\begin{array}{c}
R_{y \pm} \\
u
\end{array}\right)_{\left(x, y_{ \pm},(n+1 / 2) \Delta t\right)}
$$

Step 5. Repeat Step 1:

The results obtained at the end of Step 4 are used to solve for $\Delta t / 2$ the equations in the $\mathrm{x}$ - direction as in Step 1.

$$
\text { i.e. }\left(\begin{array}{c}
R_{x \pm} \\
v
\end{array}\right)_{(x, y,(n+1) \Delta t)}=\left(\begin{array}{c}
R_{x \pm} \\
v
\end{array}\right)_{\left(x_{ \pm}, y,(n+1 / 2) \Delta t\right)}
$$

Shoucri [11] applied the fractional steps method for the numerical solution of the shallow water equations over flat bottom. He used the calculated variables (the height and the velocity field) in studying the evolution of the potential vorticity field.

Abd-el-Malek and Helal [10] developed a mathematical simulation to determine the water velocity in the Lake Mariut, taking into consideration its concentration and the distribution of the temperature over it, by applying the fractional steps method for the numerical solution of the shallow water equations. The application they presented over flat bottom requires the variables at two time-levels $\Delta t$ and $\Delta t / 2$ according to Strang method [20] which is more accurate in time, since it has a second-order accurate. They proved the convergence of the fractional steps method and verified that the order of convergence is of the first order.

In this problem, we use the same trend as done by Abd-el-Malek and Helal [10] and Shoucri [11] in solving numerically the two-dimensional nonlinear shallow water equations in the presence of Coriolis force and bottom topography using the fractional steps method. We study the effects of the Coriolis force and the bottom topography for particular initial flows on the velocity compo- nents and the free surface elevation.

\subsection{Cubic Spline Interpolation}

To approximate the arbitrary functions on closed intervals by the aid of the polynomials, we used the most common piecewise polynomial approximation using cubic polynomials between each successive pairs of nodes which is called cubic spline interpolation. A general cubic polynomial involves four constants, so, there is a sufficient flexibility in the cubic spline procedure to ensure that the interpolant is not only continuously differentiable on the interval, but also it has a continuous second derivative on the interval, [21].

\section{Results and Discussion}

We apply the numerical scheme presented in Section 3 to solve the problem at different values of time for different initial flows describing the dam break wave and the tsunami propagation wave over two main profiles of the non-flat bottom in cases of neglecting and including the Coriolis force. We represent the bottom with two different shapes which are a hump and rising hill topography respectively, as follows.

Hump topography: (25)

Rising up hill topography: (26)

The two forms of $\zeta(x)$ are defined for $y \in[0,100]$. We present the bottom topographies considered by a hump with maximum height $250 \mathrm{~m}$ and rising hill topography up to $1850 \mathrm{~m}$, Figures 2 and 3. The problem is solved for $g=9.806 \mathrm{~m} / \mathrm{s}^{2}$ and maximum depth $H=2 \mathrm{~km}$. The scale is based with length $x=500 \mathrm{~km}$, and width $y=100 \mathrm{~km}$. The horizontal grid cell length is $\Delta x$ $=\Delta y=1 \mathrm{~km}$ resulting in $500 \times 100$ grid cells at time step $\Delta t=1 \mathrm{~s}$. We assume the initial velocities $u(x$, $y, 0)=v(x, y, 0)=0$.

The initial flows describing the tsunami propagation wave and the dam break wave are illustrated as follows:

$$
\begin{aligned}
& \zeta(x)=\left\{\begin{array}{lr}
0.125\left[1+\cos \left(\frac{\pi}{25}(x-375)\right)\right], & 350 \leq x \leq 400 \\
0 & \text { otherwise }
\end{array}\right. \\
& \zeta(x)=\left\{\begin{array}{lr}
0.925\left(1+\cos \left(\frac{\pi}{200}(x-400)\right)\right), & 200 \leq 400 \leq x \\
1.850, & \text { otherwise } \\
0 & \text { ot }
\end{array}\right.
\end{aligned}
$$




\section{Tsunami propagation wave:}

Tsunamis are long waves generated by submarine earthquakes. Once they reach the open ocean and travel through deep water tsunamis which have extremely small amplitudes but travel fast. Tsunami propagation velocity can be estimated by using the wave speed equation $C=\sqrt{g H}$. For $2000 \mathrm{~m}$ water depth, the speed will be about $504 \mathrm{~km} /$ hour. The most difficult phase of the dynamics of tsunami waves deals with their breaking as they approach the shore. This phase depends greatly on the bottom bathymetry. As a model of this initial tsunami displacement, we consider the wave presented by George [7] which is given by (27).

Dam break wave:

The dam-break problem is an environmental problem

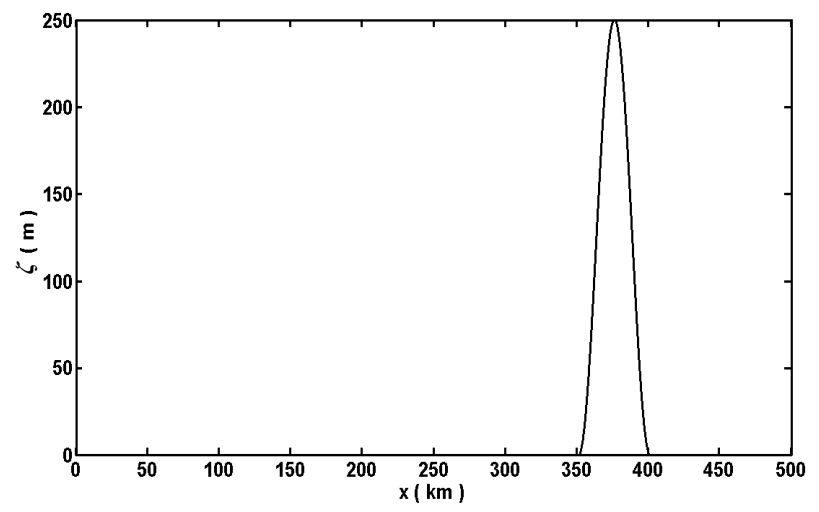

Figure 2. Profile of hump topography.

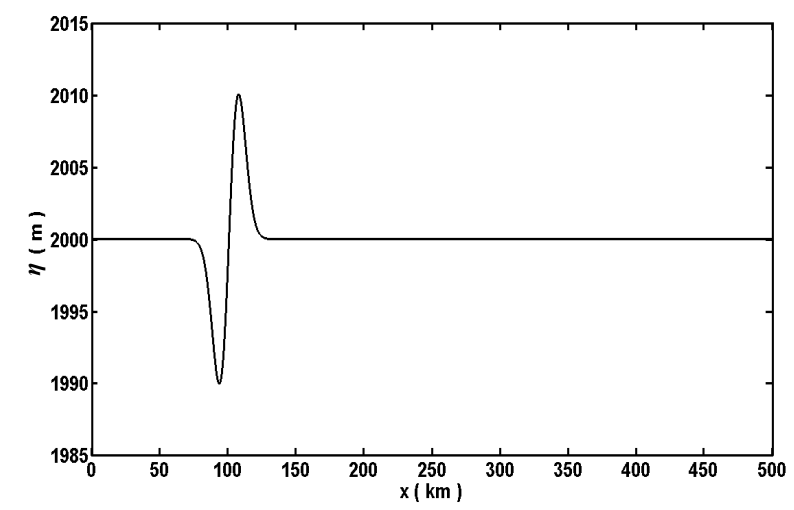

Figure 4. Tsunami propagation initial wave. involving unsteady flows in waterways. The study of flooding after the dam break is very important because of the risk to life and property in the potentially inundated area below the dam. The initial dam break model assumed is given by (28).

Figures 4 and 5 represent the tsunami propagation and dam break waves, respectively, as initial flows. In case of the dam break problem, the water is assumed to be at rest on both sides of the dam initially. At $t=0$ the dam is suddenly destroyed, causing a shock wave (bore) travelling downstream with $\eta=-10$ on $\mathrm{x}>250$ and a rarefaction wave (depression wave) traveling upstream with $\eta=10$ on $\mathrm{x}<250$. The water pushing down from above acts somewhat like a piston being pushed downstream with acceleration.

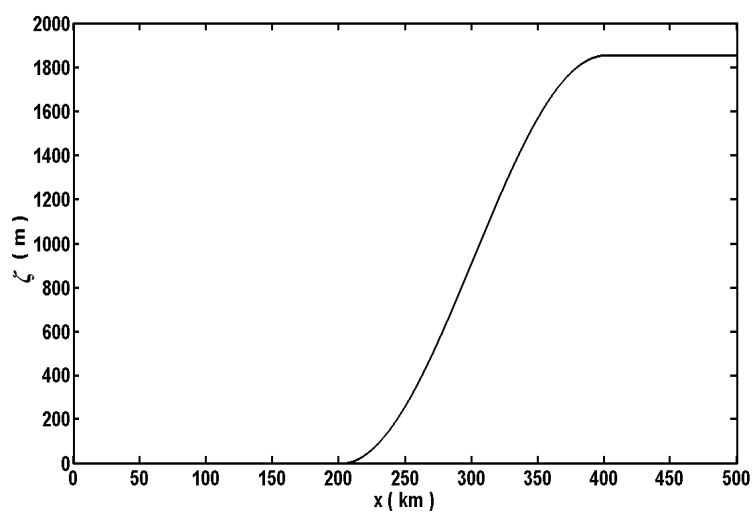

Figure 3. Profile of rising up hill topography.

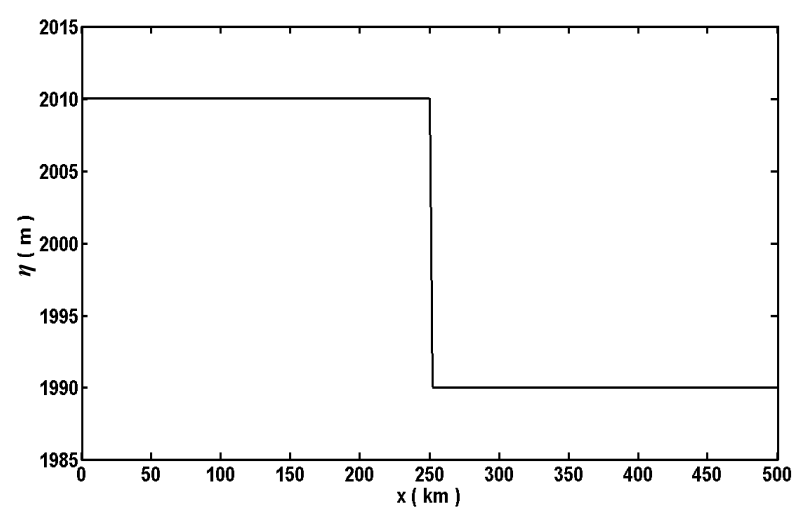

Figure 5. Dam break initial wave.

$$
\begin{aligned}
h^{*}(x, y, 0) & =\left\{\begin{array}{cc}
0.00235(x-100) e^{-\left(\frac{x-100}{10}\right)^{2}}+H, & 50 \leq x \leq 150 \\
\mathrm{H}, & \text { otherwise }
\end{array}\right. \\
h^{*}(x, y, 0) & =\left\{\begin{array}{cc}
(H+0.01) \operatorname{sgn}(x-250) & \text { for } \quad 0 \leq x \leq 250 \\
(H-0.01) \operatorname{sgn}(x-250) & \text { for } \quad 250 \leq x \leq 500
\end{array}\right.
\end{aligned}
$$


From the geophysical fluid dynamics point of view, as a first step toward understanding the role of bottom topography, consider the flow in a periodic zonal channel with solid boundaries to the North and the South with idealized topography. Here $f=0.01$ is chosen such that the domain is resides in the northern hemisphere of the earth. Sea surface velocities and water height over the bottom topography at various time and different initial conditions are illustrated in the cases of neglecting and including the Coriolis force

Equations (1)-(3) contain the most fundamental balances of shallow water flows, see [22,23]. The convective part on the left-hand side is a hyperbolic system of conservation laws and the source term on the right-hand side is due to gravitational acceleration and rotation of the earth (Coriolis force). The steady state results from a balance between the advection and decay processes [24].

This suggests that we may have difficulties with a fractional-step method in order to balance between the advection terms and the source terms, where we first solve the advection equation ignoring the reactions and then solve the reaction equation ignoring the advection [24]. Even if we start with the exact steady-state solution, each of these steps can be expected to make a change in the solution. In principle the two effects should exactly cancel out, but numerically they typically will not, since different numerical techniques are used in each step [24].

\subsection{The Velocity Component in the $x$-Direction}

Figures 6 and 7 illustrate the behavior of the velocity $u$ over the topography $\zeta(x)$ represented by a hump given by (25) for an initial flow represented by the dam break wave given by (28), in case of neglecting and including the Coriolis force at $t=400,1200$ and 1800 sec respectively. By neglecting the effect of earth rotation (i.e. no Coriolis force), the coupling between Equations (2) and (3) due to Coriolis force does no longer exists. Consequently, it is expected that the velocity vector is in the direction of wave propagation and that minor oscillations will appear in the results when the wave propagates

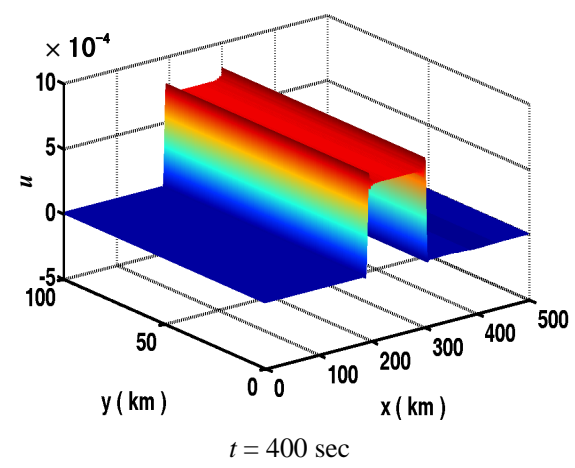

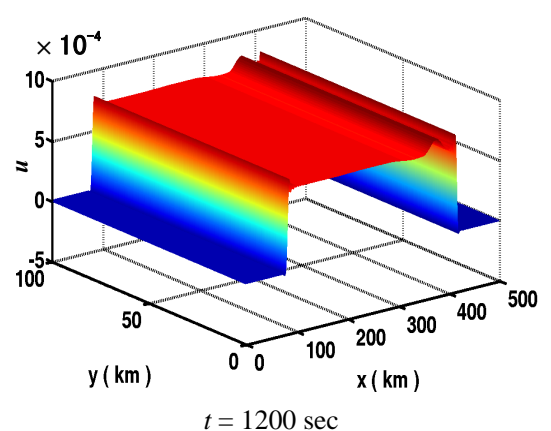

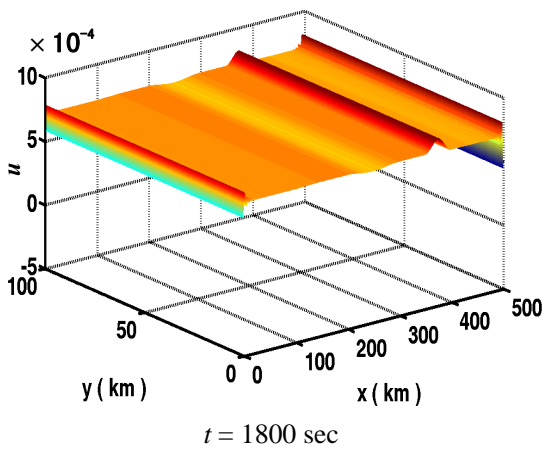

Figure 6. Behavior of $u$ with hump topography and dam break initial flow without the effect of the Coriolis force at $t$ $=400,1200$ and $1800 \mathrm{sec}$.

over topography. This is in good agreement with the result shown in Figure 6. In fact, since the water at $x=$ $250 \mathrm{~km}$ seems likely to acquire instantaneously, a velocity different from zero, Figure 6.
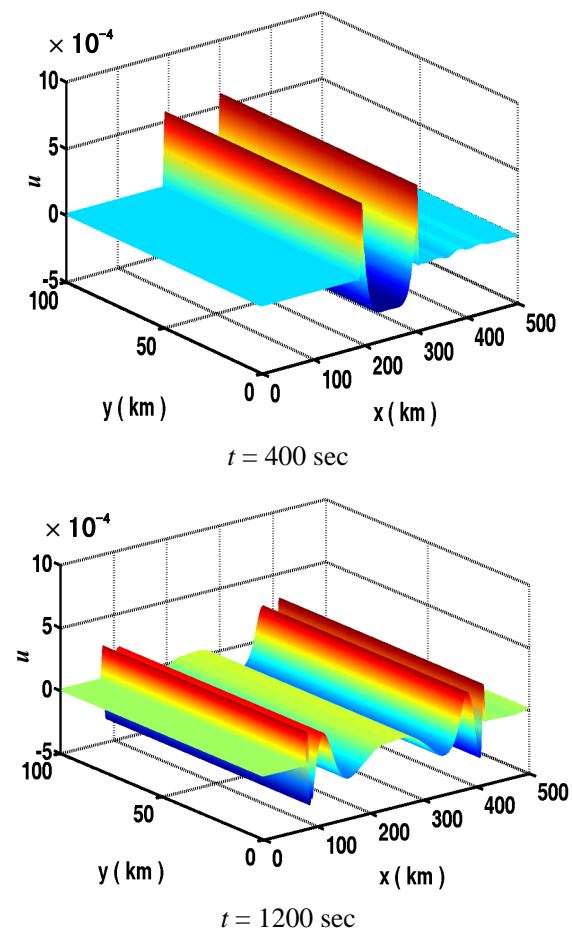


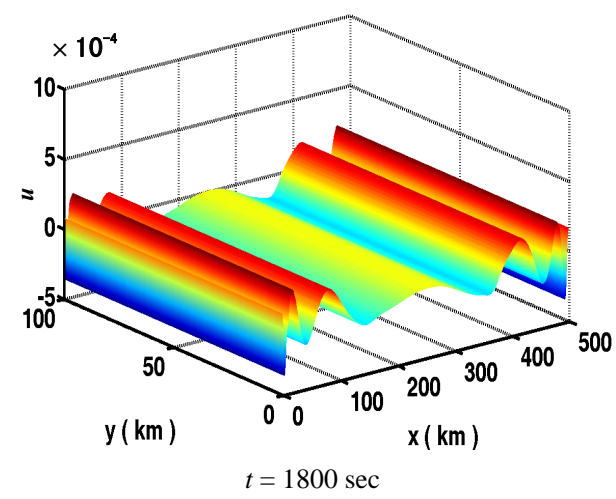

Figure 7. Behavior of $\boldsymbol{u}$ with hump topography and dam break initial flow with the effect the Coriolis force at $t=400$, 1200 and 1800 sec.

Figures 8 and 9 illustrate the behavior of the velocity $u$ over the topography $\zeta(x)$ representing a rising up function given by (26) for an initial flow representing the tsunami propagation wave given by (27), in case of neglecting and including the Coriolis force at $t=400,1200$ and $3000 \mathrm{sec}$ respectively. As mentioned before, in case of neglecting the Coriolis force, the velocity vector is in the direction of the wave propagation and minor oscillations will appear in the results when the wave propagates over the topography, Figure 8.
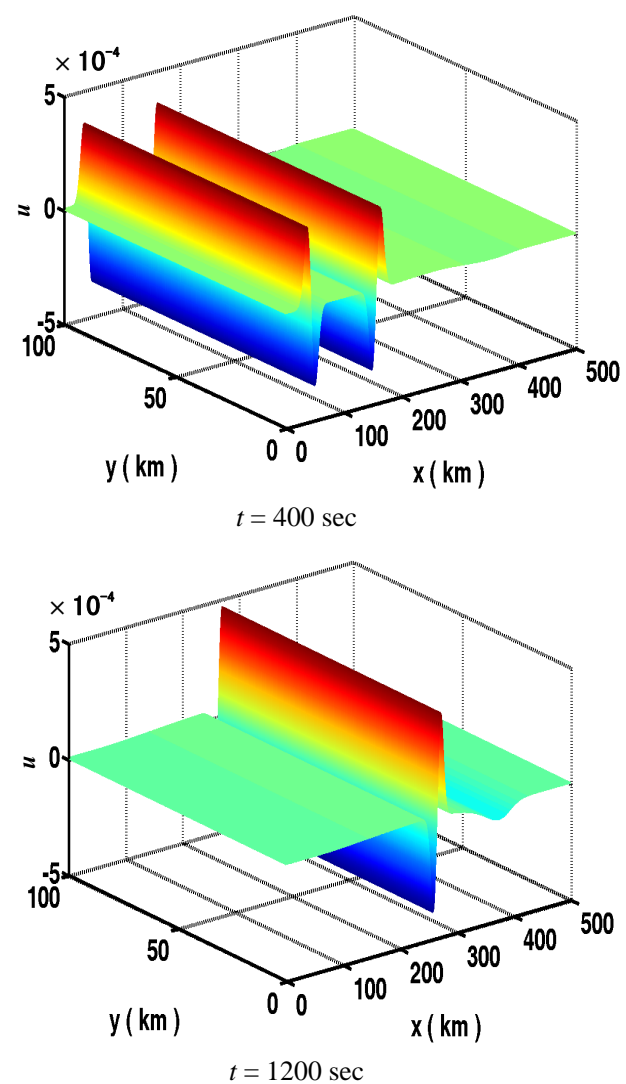

Copyright (C) 2010 SciRes.

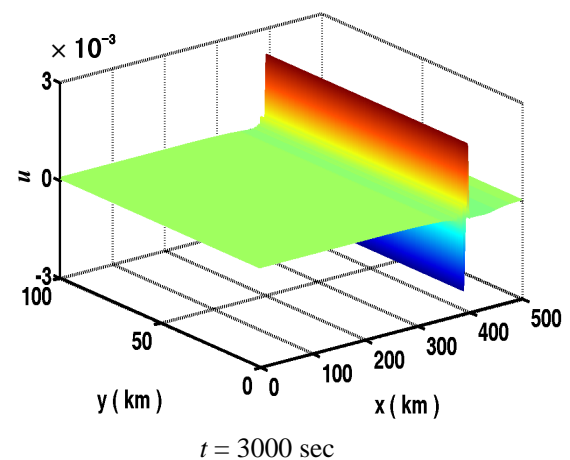

Figure 8. Behavior of $u$ with rising up topography and tsunami propagation initial flow without the effect of the Coriolis force at $t=400,1200$ and 3000 sec.
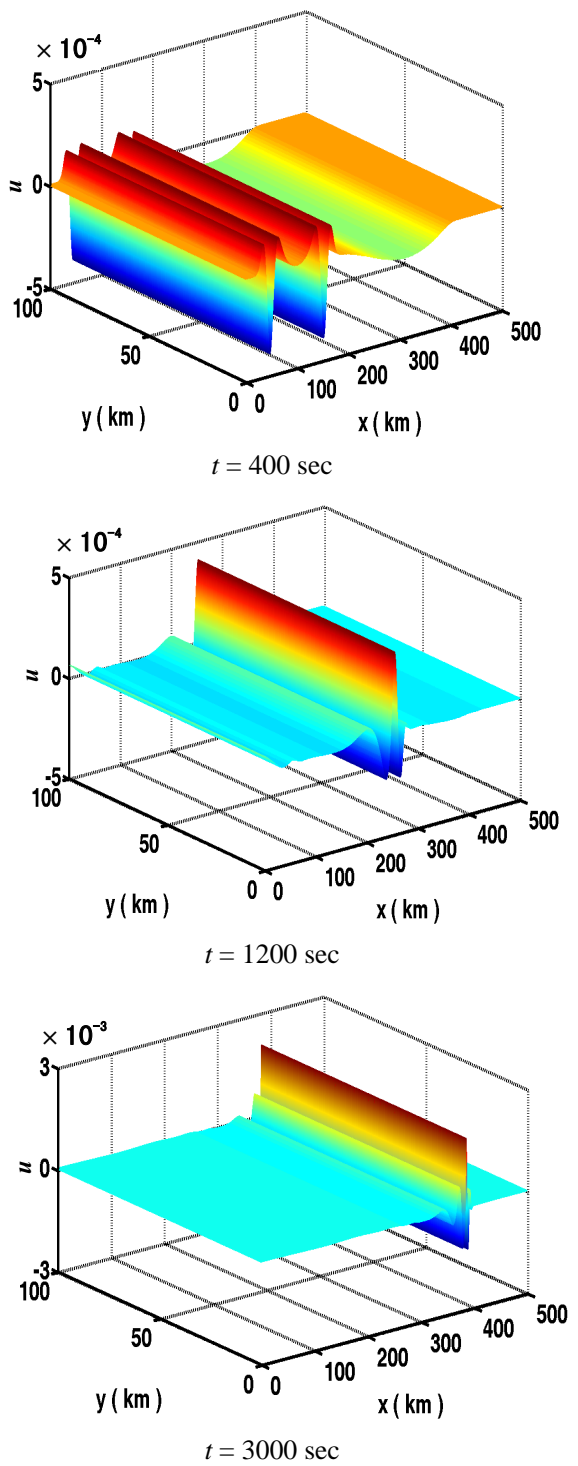

Figure 9. Behavior of $u$ with rising up topography and tsunami propagation initial flow with the effect the Coriolis force at $t=400,1200$ and $3000 \mathrm{sec}$. 


\subsection{The Velocity Component in the y-Direction}

Figures 10 and 11 illustrate the behavior of the velocity $v$ over the topography $\zeta(x)$ representing a hump given by (25) for an initial flow representing the dam break wave give $n$ by (28) and a rising up function given by (26) for an initial flow representing the tsunami propagation wave given by (27) with the effect of Coriolis force.

\subsection{The Water Height $\boldsymbol{h}^{*}$}

Figures 12 and 13 illustrate the behavior of the water height $h^{*}$ over the topography $\zeta(x)$ representing by a hump given by (25) for an initial flow representing by

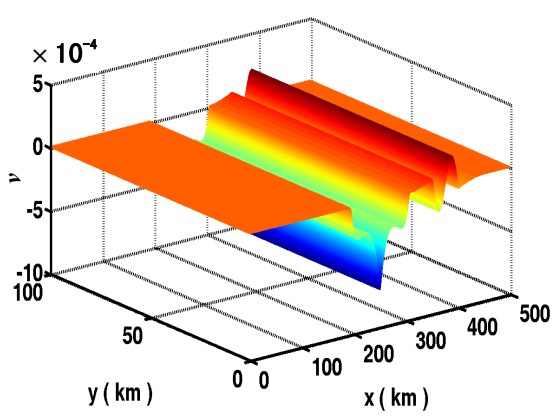

$t=400 \mathrm{sec}$

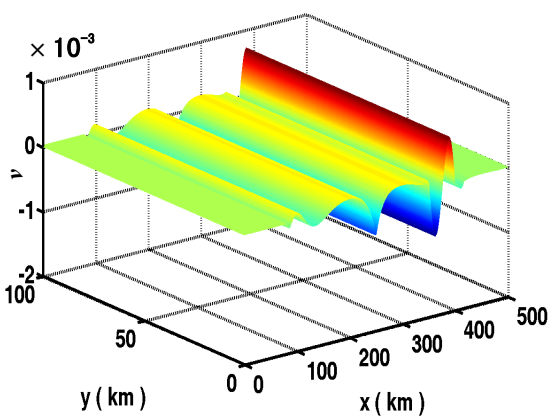

$t=1200 \mathrm{sec}$

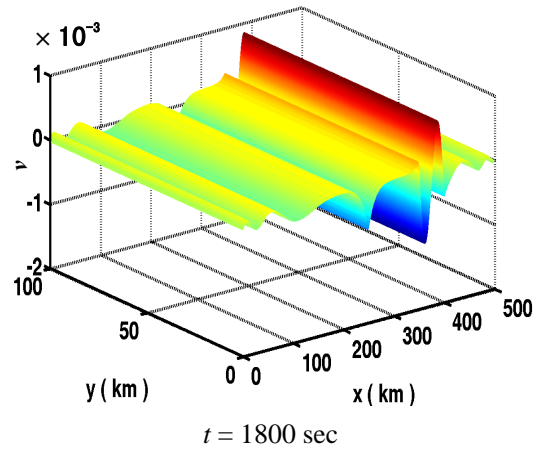

Figure 10. Behavior of $v$ with hump topography and dam break initial flow with the effect of the Coriolis force at $\boldsymbol{t}=$ 400,1200 and 1800 sec. the dam break wave given by (28) in case of neglecting and including the Coriolis force at $t=400,1200$ and 1800 sec respectively at $\mathrm{y}=0$.

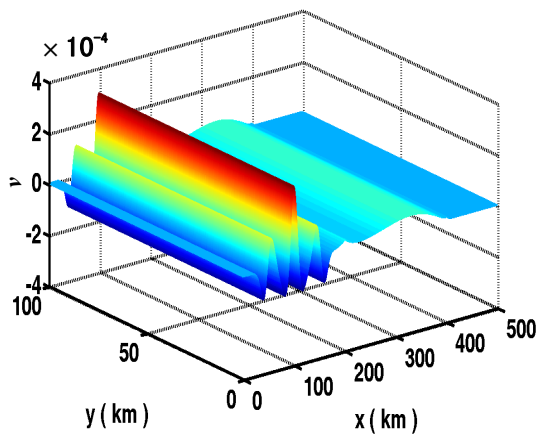

$t=400 \mathrm{sec}$

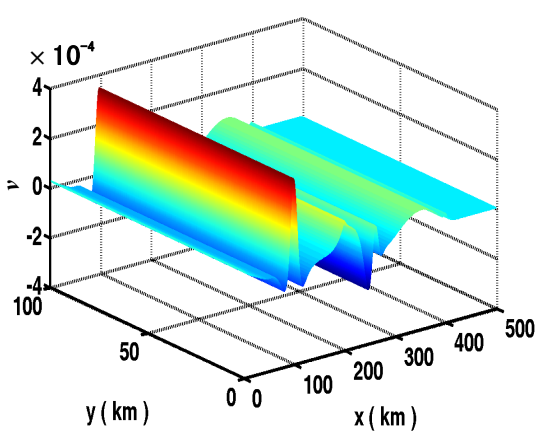

$t=1200 \mathrm{sec}$

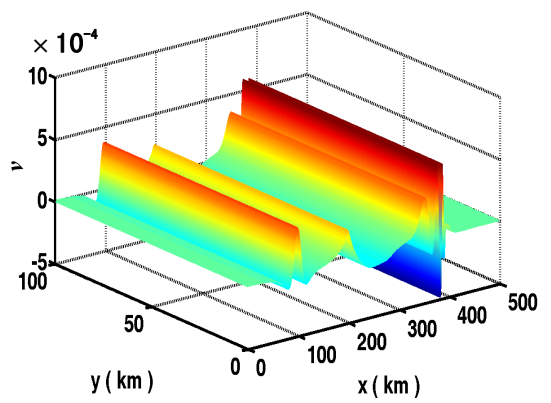

$t=3000 \mathrm{sec}$

Figure 11. Behavior of $v$ with rising up topography and tsunami propagation initial flow with the effect the Coriolis force at $t=400,1200$ and 3000 sec.

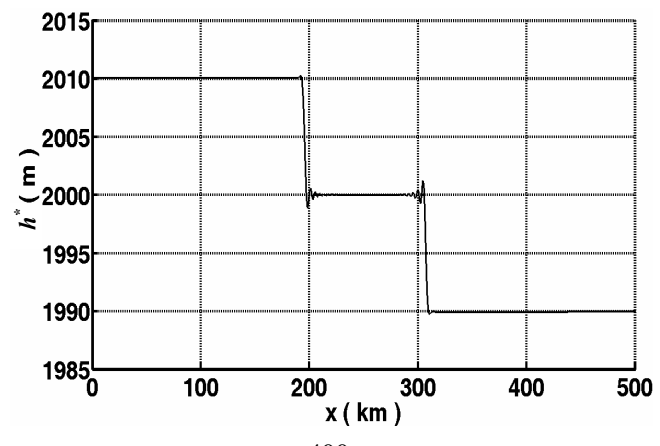

$t=400 \mathrm{sec}$ 


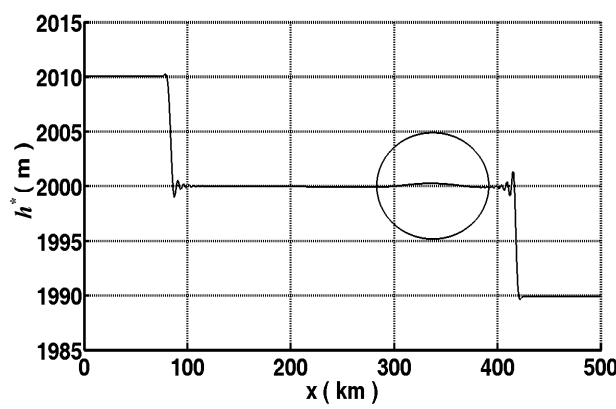

$t=1200 \mathrm{sec}$

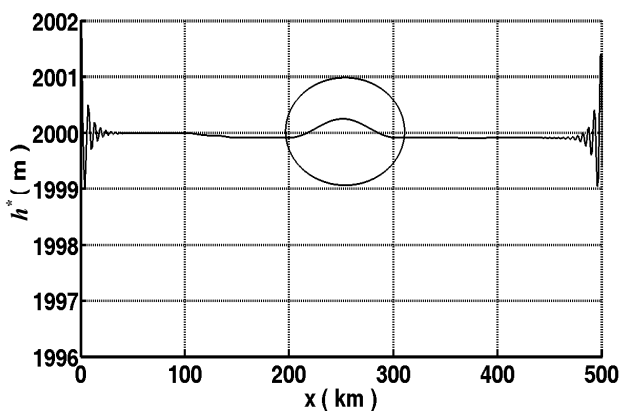

$t=1800 \mathrm{sec}$

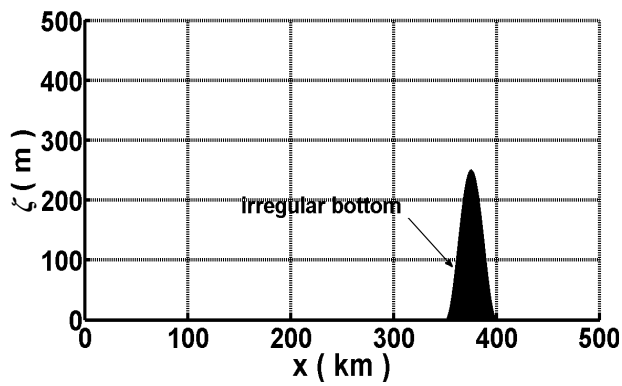

Figure 12. Behavior of $h^{*}$ with hump topography and dam break initial flow without the effect of the Coriolis force at $t$ $=400,1200$ and $1800 \mathrm{sec}$.

Figures 14 and 15 illustrate the behavior of the water height $h^{*}$ over the topography $\zeta(x)$ representing a rising up function given by (26) for an initial flow representing by the tsunami propagation wave given by (27) in case of neglecting and including the Coriolis force at $t$ $=400,1200$ and $3000 \mathrm{sec}$ respectively at $\mathrm{y}=0$.

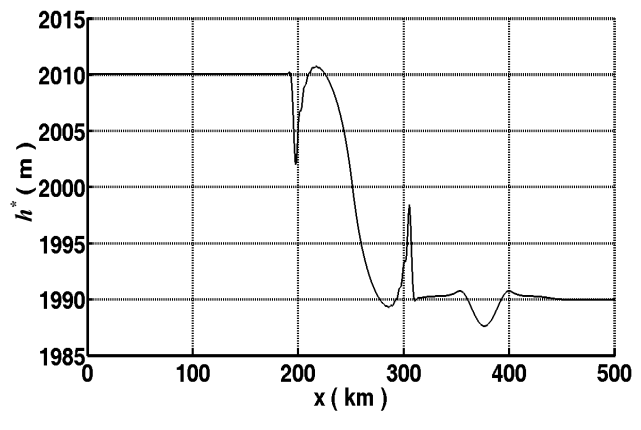

$t=400 \mathrm{sec}$

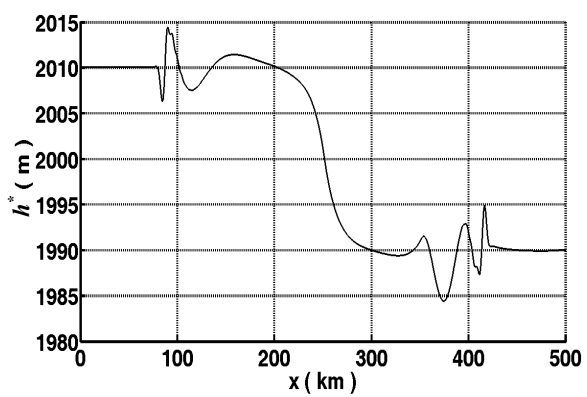

$t=1200 \mathrm{sec}$

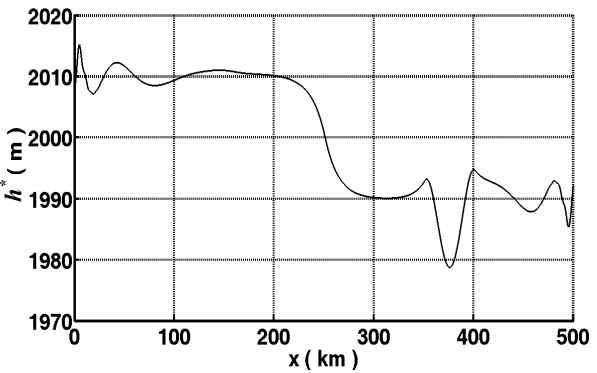

$t=1800 \mathrm{sec}$

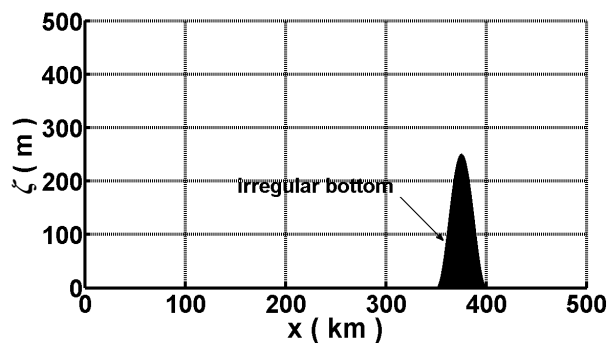

Figure 13. Behavior of $h^{*}$ with hump topography and dam break initial flow with the effect the Coriolis force at $t=400$, 1200 and 1800 sec.

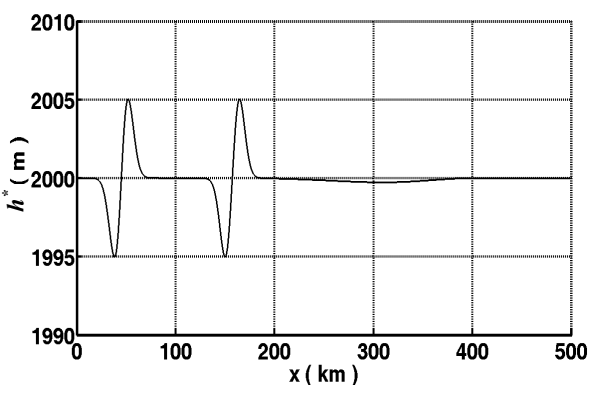

$t=400 \mathrm{sec}$

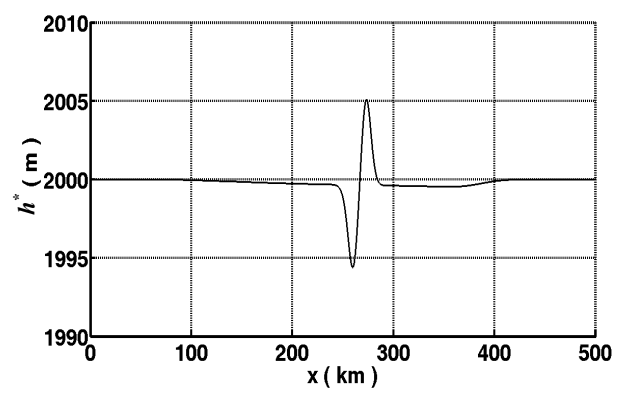

$t=1200 \mathrm{sec}$ 

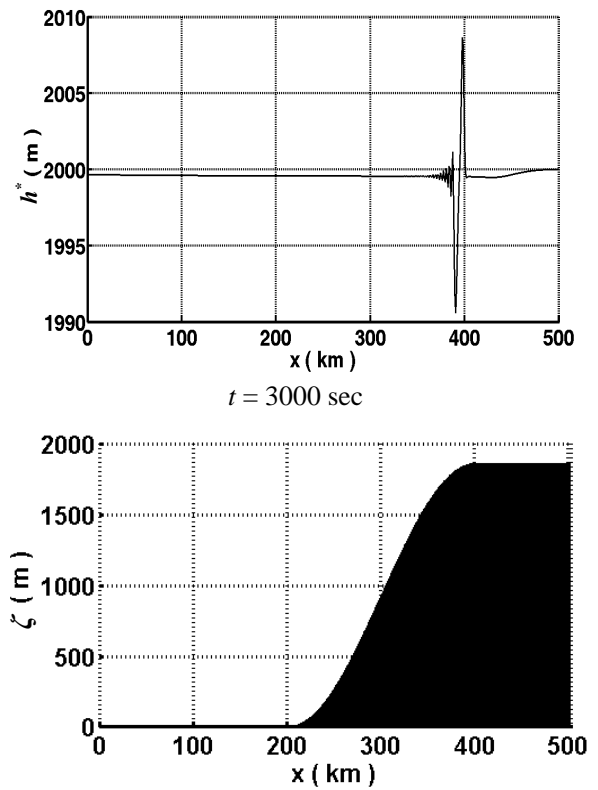

Figure 14. Behavior of $h^{*}$ with rising up topography and tsunami propagation initial flow without the effect of the Coriolis force at $t=400,1200$ and $3000 \mathrm{sec}$.
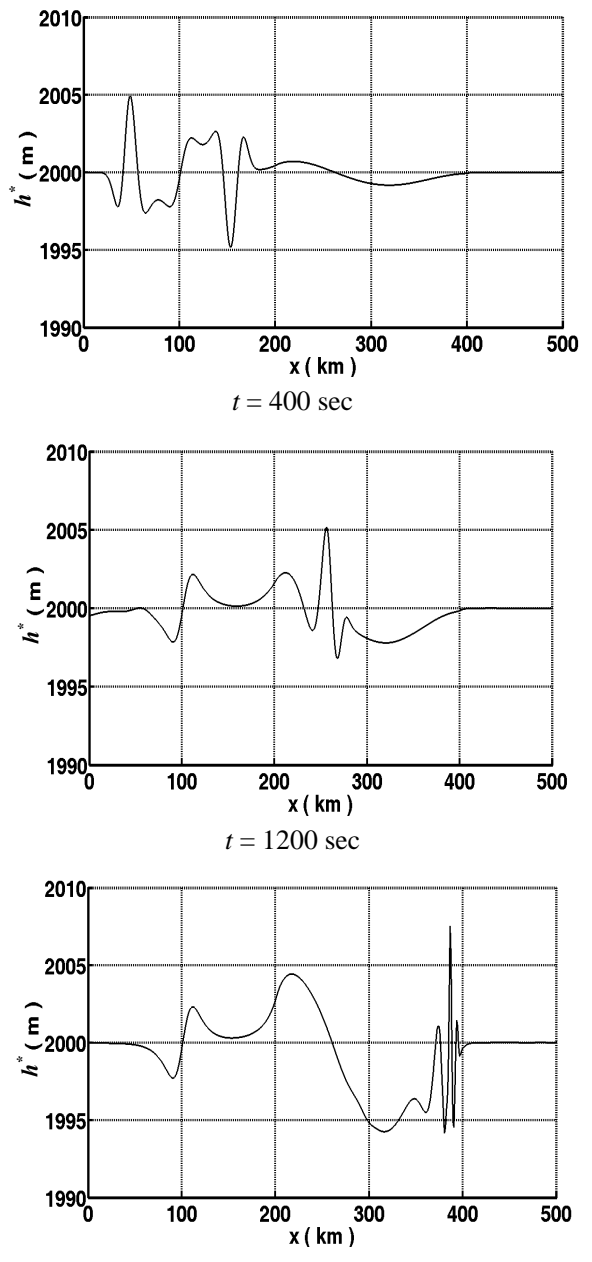

$t=3000 \mathrm{sec}$

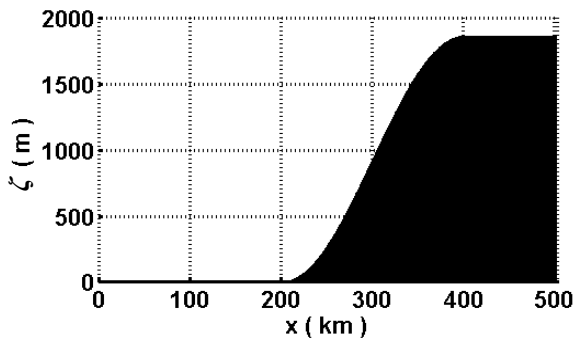

Figure 15. Behavior of $h^{*}$ with rising up topography and tsunami propagation initial flow with the effect the Coriolis force at $t=400,1200$ and $3000 \mathrm{sec}$.

Since the water at $x=250 \mathrm{~km}$ seems likely to acquire instantaneously, it is plausible that a shock would be created instantly on the upstream side and a relatively small propagated wave in the negative X-direction due to bottom topography as shown in the circle in Figure 12.

By taking the Coriolis force $(f v,-f u)$ into consideration, it becomes responsible for the oscillatory motion according to the solutions of Equations (23) and (24) obtained in Step 3 in the fractional steps method. The coupling between the velocity components $u$ and $v$ causes the deflection of fluid parcels which are oscillating back and forth in the direction of wave motion and causes gravity waves to disperse as shown in Figures 13 and 15. The magnitude of this deflection proved to be independent of the absolute depth and depends only on the slope of the bottom, as demonstrated in the momentum Equations (2) and (3).

In case of the tsunami wave, the initial waves split into two similar waves, one propagates in the positive $\mathrm{x}$-direction and the other in the negative $x$-direction. The height above mean sea level of the two oppositely traveling tsunamis is approximately half that of the original tsunami, as shown in Figure 14. This happened because the potential energy that results from pushing water above mean sea level is transferred to horizontal propagation of the tsunami wave (kinetic energy) (i.e. the tsunami converts potential energy into kinetic energy). It is well known when the local tsunami travels over the continental topography, that the wave amplitude increases and the wavelength and velocity decreases, which results in steepening of the leading wave, see Figure 14.

In case of the dam break problem, the effect of the Coriolis force on the water height at a sequence of times after the breakage of a dam causes a wave travelling downstream and a wave travelling upstream as shown in Figure 13. The rarefaction wave that developed in Figure 13 is overtaken by dispersive wave which should form shocks on both sides of propagation.

In case of the tsunami waves, they propagate in coherent wave packets, with little loss of amplitude over very long distances as shown in Figure 15. As the water depth decreases, the wave amplitude increases and the wavelength and velocity decreases, resulting in steepening of the dispersed wave. 


\subsection{The Free Surface Elevation ( $\eta$ )}

Figures 16 and 17 illustrate the effect of the Coriolis force on the free surface elevation $\eta$ when travelling over the topography $\zeta(x)$ representing a hump given by (25) and a rising up function given by (26) for an initial flow representing the dam break wave given by (28) at $t=1200 \mathrm{sec}$.

The 500 kilometers long dam wall, which runs parallel to the $y$-axis is 10 meters wide and is centered at $x=250$ kilometers. Along the boundaries, at $\mathrm{x}=0$ kilometers and $\mathrm{x}=500$ kilometers and $\eta$ is fixed at the upstream and downstream water depth respectively. All other boundaries are considered as reflective boundary conditions. In case of neglecting the Coriolis force, a shock front always exists as shown in Figure 16, while the free surface elevation $\eta$ under the effect of the Coriolis force has no shock travelling downstream and hence travelled in the direction of propagation over the topography $\zeta(x)$ as seen in Figure 17.

As seen in Figure 16, the jump at the generated shock first decreases and then it increases as the shock approaches the bottom topography. It is observed that the increasing in the shock wave when travelling over the rising up hill topography is greater than when travelling over hump topography.

It can be observed that the free surface elevation $\eta$ in Figure 17 clearly differs from that in Figure 16 due to the effect of the Coriolis force which is responsible for the oscillatory motion in the direction of wave motion which causes gravity waves to disperse.

Figures 18 and 19 illustrate the effect of the Coriolis force on the free surface elevation $\eta$ when travelling over the topography $\zeta(x)$ representing a hump given by (25) and a rising up function given by (26) for an initial flow representing the tsunami propagation wave given by (27), at $t=2400 \mathrm{sec}$.

The effect of the Coriolis force on the tsunami propagation wave is responsible for some part of the energy, which is transmitted to the ocean with the seismic bottom motions, to accumulate in the region of the disturbance. This leads to a reduction of the barotropic wave energy and tsunami amplitude. The direction of the tsunami radiation varies and the energy flow transferred by the waves is redistributed.

The effect of Coriolis force on transoceanic tsunami with and without Coriolis terms shows differences in wave height but not much difference in arrival time as observed in Figure 18 and Figure 19.

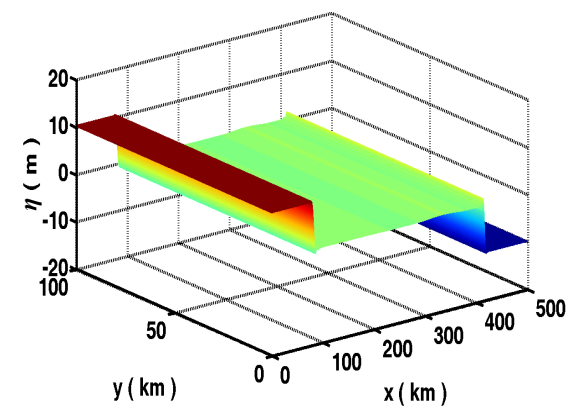

(a)

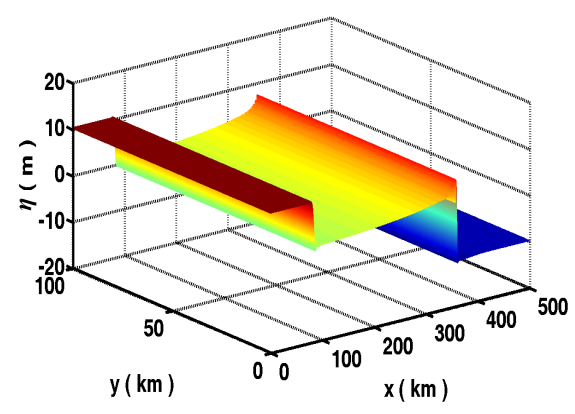

(b)

Figure 16. Behavior of $\eta$ for the dam break initial flow without the effect of the Coriolis force at $t=1200$ sec over: (a) Hump topography; (b) Rising up hill topography.

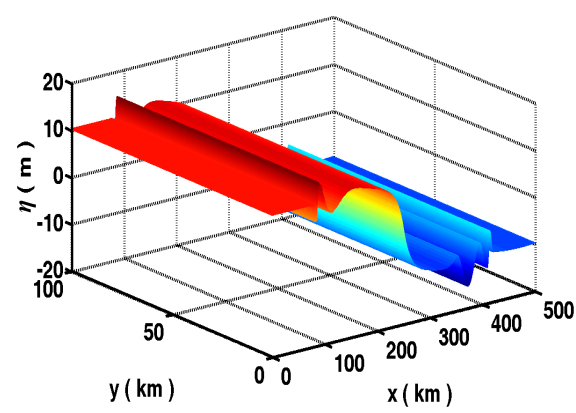

(a)

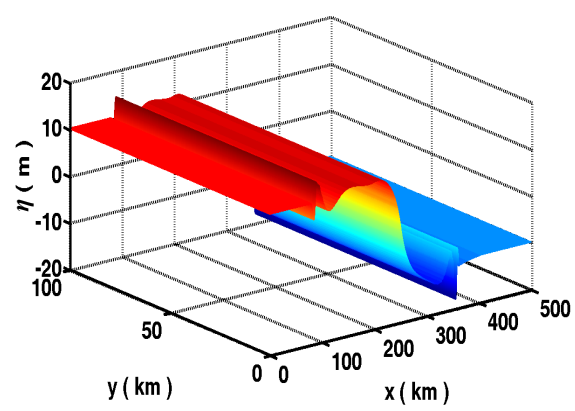

(b)

Figure 17. Behavior of $\eta$ for the dam break initial flow with the effect of the Coriolis force at $t=1200$ sec over: (a) Hump topography; (b) Rising up hill topography. 


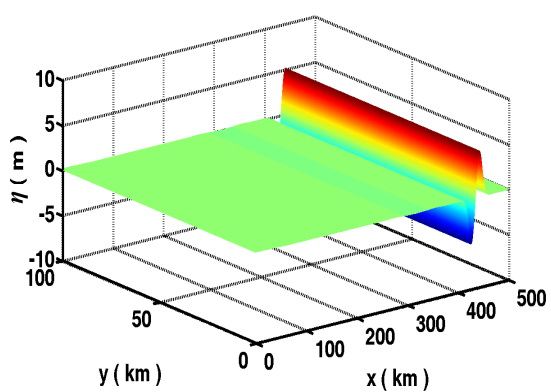

(a)

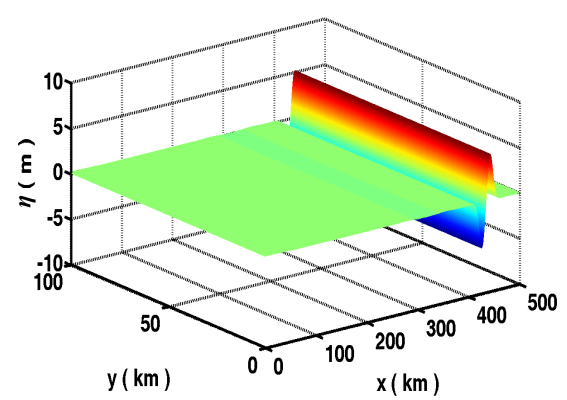

(b)

Figure 18. Behavior of $\eta$ for the tsunami propagation initial flow without the effect of the Coriolis force at $t=2400$ sec over: (a) Hump topography; (b) Rising up hill topography.



(a)

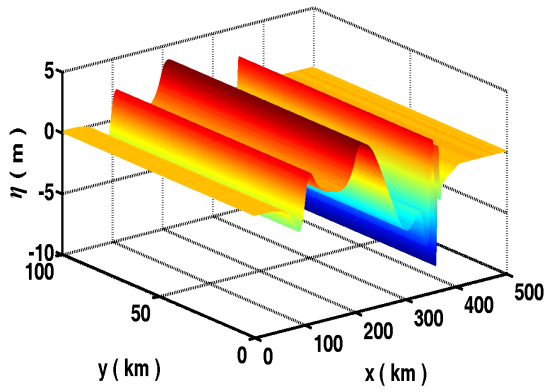

(b)

Figure 19. Behavior of $\eta$ for the tsunami propagation initial flow with the effect of the Coriolis force at $t=2400$ sec over: (a) Hump topography; (b) Rising up hill topography.

Therefore the dispersion effects become more significant as the wave energy is more spatially spread out and scattered. The variability of the bathymetry is also quite variable being an important parameter of wave dispersion as it controlled in the speed and the height of the tsunami wave when there was a sudden change in the depth of water as seen in Figure 18 and Figure 19.

As seen from Figures 16-19, the wave propagated faster when travelling over the hump topography than travelling over the increasing hill topography. This was expected due to the wave speed equation $C=\sqrt{g H}$ which is proportional to the water depth and inversely proportional to the bottom bathymetry.

\section{Conclusions}

The fractional steps method for the numerical solution of the shallow water equations is applied to study the evolution of the height and the velocity field of the flow under the effect of Coriolis force and bottom topography. The method consists of splitting the equations and successively integrating in the $\mathrm{x}$-and $\mathrm{y}$-directions along the characteristics using the Riemann invariants, associated with the cubic spline interpolation. In this work, we applied the fractional steps method to solve the two-dimensional shallow water equations with source terms (in- cluding the Coriolis force and bottom topography) for two different initial flows namely dam break wave and tsunami wave. The presence of the Coriolis force in the shallow water equations causes the deflection of fluid parcels in the direction of wave motion and causes gravity waves to disperse. As water depth decreases due to bottom topography, the wave amplitude increases, the wavelength and wave speed decreases resulting in steepening of the wave. The effect of the Coriolis force is responsible for the oscillatory motion in the direction of wave motion which causes gravity waves to disperse. The overall performance of the fractional steps method in solving the shallow water equations with source terms is particularly attractive, simple, efficient and highly accurate as our results verified the reality about the nature of the dam break problem and the tsunami propagation wave. In future, we shall apply the fractional steps method to the shallow water equations with the source term including Coriolis force and a movable topography as it appears in oceanographic modeling as well as in the river flow modeling is underway and compare the results analytically using Lie-group method, [25-29].

\section{Acknowledgements}

The authors would like to express their sincere thank the 
reviewers for suggesting certain changes in the original manuscript, for their valuable comments which improved the paper and for their great interest in that work.

\section{References}

[1] D. G. Dritschel, L. M. Polvani and A. R. Mohebalhojeh, "The Contour-Advective Semi-Lagrangian Algorithm for the Shallow Water Equations," Monthly Weather Review, Vol. 127, No. 7, 1999, pp. 1551-1564.

[2] J. Pedlosky, “Geophysical Fluid Dynamics," Springer, New York, 1987.

[3] M. Lukácová-Medvid'ová, S. Noelle and M. Kraft, "WellBalanced Finite Volume Evolution Galerkin Methods for the Shallow Water Equations," Journal of Computational Physics, Vol. 221, No. 1, 2007, pp. 122-147.

[4] T. Gallouët, J. M. Hérard and N. Seguin, "Some Approximate Godunov Schemes to Compute Shallow-Water Equations with Topography," Computers \& Fluids, Vol. 32, No. 4, 2003, pp. 479-513.

[5] P. J. Dellar and R. Salmon, "Shallow Water Equations with a Complete Coriolis Force and Topography," Physics of Fluids, Vol. 17, No. 10, 2005, pp. 106601-106619.

[6] K. V. Karelsky, V. V.Papkov, A. S. Petrosyan and D. V. Tsygankov, "Particular Solution of the Shallow-Water Equations over a Non-Flat Surface," Physics Letters A, Vol. 271, No. 5-6, 2000, pp. 341-348.

[7] D. L. George, “Augmented Riemann Solvers for the Shallow Water Equations over Variable Topography with Steady States and Inundation,” Journal of Computational Physics, Vol. 227, No. 6, 2008, pp. 3089-3113.

[8] M. Shoucri, "The Application of a Fractional Steps Method for the Numerical Solution of the Shallow Water Equations," Computer Physics Communications, Vol. 164, No. 1-3, 2004, pp. 396-401.

[9] A. Stainiforth and C. Temperton, "Semi-Implicit SemiLagrangian Integration Scheme for a Baratropic FiniteElement Regional Model,” Monthly Weather Review, Vol. 114, No. 11, 1986, pp. 2078-2090.

[10] M. B. Abd-el-Malek and M. H. Helal, "Application of the Fractional Steps Method for the Numerical Solution of the Two-Dimensional Modeling of the Lake Mariut," Applied Mathematical Modeling, Vol. 33, No. 2, 2009, pp. 822-834.

[11] M. Shoucri, "Numerical Solution of the Shallow Water Equations with a Fractional Step Method," Computer Physics Communications, Vol. 176, No. 1, 2007, pp. 23-32.

[12] I. Yohsuke, A. Takayuki and M. Shoucri, "Comparison of Efficient Explicit Schemes for Shallow-Water EquationsCharacteristics-Based Fractional-Step Method and Multimoment Eulerian Scheme,” Journal of Applied Meteorology and Climatology, Vol. 46, No. 3, 2007, pp. 388-395.

[13] A. Takayuki, "Interpolated Differential Operator (IDO) Scheme for Solving Partial Differential Equations," Computer Physics Communications, Vol. 102, No. 1-3, 1997, pp. 132-146.

[14] C. Temperton and A. Stainiforth, "An Efficient TwoTime-Level Semi-Lagrangian Semi-Implicit Integration Scheme,” Quarterly Journal of the Royal Meteorological
Society, Vol. 113, No. 477, 1987, pp. 1025-1039.

[15] E. Audusse, F. Bouchut, M. Bristeau, R. Klein and B. Perthame, "A Fast and Stable Well-Balanced Scheme with Hydrostatic Reconstruction for Shallow Water Flows," SIAM Journal on Scientific Computing, Vol. 25, No. 6, 2004, pp. 2050-2065.

[16] M. E. Talibi and M. H. Tber, "On a Problem of Shallow Water Type," Electronic Journal of Differential Equations, Vol. 11, 2004, pp. 109-116.

[17] V. R. Ambati and O. Bokhove, "Space-Time Discontinuous Galerkin Discretization of Rotating Shallow Water Equations,” Journal of Computational Physics, Vol. 225, No. 2, 2007, pp. 1233-1261.

[18] N. N. Yanenko, "The Method of Fractional Steps, the Solution of Problems of Mathematical Physics in Several Variables,” Springer-Verlag, Berlin, 1971.

[19] D. R. Durran, "Numerical Methods for Wave Equations in Geophysics Fluid Dynamics," Springer-Verlag, New York, 1999.

[20] G. Strang, "On the Construction and Comparison of Finite Difference Schemes,” Society for Industrial and Applied Mathematics, Journal for Numerical Analysis, Vol. 5, No. 3, 1968, pp. 506-517.

[21] R. L. Burden and J. D. Faires, "Numerical Analysis," PWS Publishing Company, Boston, 1993.

[22] S. Noelle, N. Pankratz, G. Puppo and J. R. Natvig, "Well-Balanced Finite Volume Schemes of Arbitrary Order of Accuracy for Shallow Water Flows," Journal of Computational Physics, Vol. 213, No. 2, 2006, pp. 474-499.

[23] S. Noelle, Y. Xing and C. Shu, "High-Order Well-Balanced Finite Volume WENO Schemes for Shallow Water Equation with Moving Water,” Journal of Computational Physics, Vol. 226, No. 1, 2007, pp. 29-58.

[24] R. LeVeque, "Finite Volume Methods for Hyperbolic Problems,” Cambridge University Press, Cambridge, 2004.

[25] Y. Z. Boutros, M. B. Abd-el-Malek, N. A. Badran and H. S. Hassan, "Lie-Group Method for Unsteady Flows in a Semi-infinite Expanding or Contracting Pipe with Injection or Suction through a Porous Wall," Journal of Computational and Applied Mathematics, Vol. 197, No. 2, 2006, pp. 465-494.

[26] Y. Z. Boutros, M. B. Abd-el-Malek, N. A. Badran and H. S. Hassan, "Lie-Group Method Solution for Two-dimensional Viscous Flow between Slowly Expanding or Contracting Walls with Weak Permeability,” Applied Mathematical Modelling, Vol. 31, No. 6, 2007, pp. 1092-1108.

[27] Y. Z. Boutros, M. B. Abd-el-Malek, N. A. Badran and H. S. Hassan, "Lie-Group Method of Solution for Steady Two-Dimensional Boundary-Layer Stagnation-Point Flow Towards a Heated Stretching Sheet Placed in a Porous Medium,” Meccanica, Vol. 41, No. 6, 2007, pp. 681-691.

[28] M. B. Abd-el-Malek, N. A. Badran and H. S. Hassan, "Lie-Group Method for Predicting Water Content for Immiscible Flow of Two Fluids in a Porous Medium," Applied Mathematical Sciences, Vol. 1, No. 24, 2007, pp. 1169-1180.

[29] M. B. Abd-el-Malek and H. S. Hassan, "Symmetry Analysis for Solving Problem of Rivlin-Ericksen Fluid of Second Grade Subject to Suction,” submitted for publication. 